\title{
Utilização das matrizes de transição na avaliação e simulação precoces do crescimento de povoamentos de Pinus taeda $\mathbf{L}$.
}

\author{
Julio E. Arce ${ }^{1}$ \\ Walquiria Pizatto ${ }^{1}$ \\ CARlos R. S ANQuetta ${ }^{2}$ \\ JEFERSON L.G. WENDLING ${ }^{1}$ \\ ROMUALDO MAESTRI ${ }^{3}$
}

\begin{abstract}
RESUMO
É avaliado e simulado o crescimento de povoamentos jovens, equiâneos, monoespecíficos e homogêneos de Pinus taeda L. utilizando a técnica de simulação Matrizes de Transição. Os dados utilizados provêm de medições realizadas em árvores individuais em 1993 e 1996 (6ํㅜ e $9^{\circ}$ anos), em um experimento de espaçamentos em Pinus taeda L., instalado no município de Jaguariaíva - PR, na Fazenda Lageado, de propriedade da empresa "Pisa Florestal". A construção das matrizes de transição a partir de observações diamétricas obtidas de povoamentos de Pinus taeda em duas idades é factível e permite obter simulações coerentes para um período igual ao intervalo entre estas idades. Já a simulação para intervalos de tempo maiores do que o período considerado para a construção das matrizes de transição é desaconselhável, por se tratar de povoamentos jovens e não estar disponível, portanto, informação do ciclo completo da dinâmica da floresta.
\end{abstract}

Palavras chave: distribuições diamétricas, predição, espaçamentos, dinâmica do povoamento.

\footnotetext{
ABSTRACT

Use of transition matrices in precocious growth evaluation and simulation of Pinus taeda L. stands. The growth of young, even-aged, monospecific and homogeneous stands of Pinus taeda L. are evaluated and simulated with the Transition Matrix simulation technique. The used data was obtained from measurements over individual trees in 1993 and $1996\left(6^{\text {th }}\right.$ and $9^{\text {th }}$ years $)$ in an spacing test of Pinus taeda L. at farm Fazenda Lageado in Jaguariaiva-PR, from the Pisa Florestal company. The

${ }^{1}$ Engenheiro Florestal, Aluno de Mestrado em Manejo Florestal do Curso de Pós Graduação em Engenharia Florestal da UFPR.

${ }^{2}$ Engenheiro Florestal, M.Sc., Ph.D., Professor do Departamento de Silvicultura e Manejo, UFPR.

${ }^{3}$ Engenheiro Florestal, M. Sc., PISA Florestal S.A.
} 
transition matrixes construction from two diameter observations separated by a time interval are feasible and conduce to coherent simulations for a similar time interval that the used for the transition matrixes construction. Simulations for longer time intervals that the used for the transition matrixes construction are not recommended because the sampled stands are young and it is not disposable information about the complete dynamic cycle of the forest.

Key words: diameter distributions, prediction, spacing, stand dynamic.

\section{INTRODUÇÃO}

Um manejo florestal efetivo implica na aplicação de um sistema de tratamentos para o controle da floresta, de tal maneira que o incremento no valor econômico e/ou social da floresta seja maior do que os juros acumulados dos custos dos tratamentos (ALDER, 1980). O manejo silvicultural dentro de um povoamento pode ser feito árvore por árvore, ou por agrupamentos, mas o maior interesse da engenharia florestal centra-se no efeito dos tratamentos sobre o volume, o valor ou a estrutura, na totalidade do povoamento (DANIEL et allii, 1982).

O crescimento dos povoamentos puros coetâneos se vê afetado pelo estado de desenvolvimento da comunidade, a qualidade do sítio, a espécie, a densidade - em termos de área basal e do número de árvores por unidade de superfície -, os tratamentos silviculturais, e as unidades nas quais é expresso o crescimento. A densidade do povoamento é o segundo fator em importância, depois da qualidade do sítio, para a determinação da produtividade de um sítio florestal (DANIEL et allii, 1982), para uma determinada qualidade do material genético utilizado. A densidade do povoamento é o principal fator de produção que o silvicultor pode manejar durante o desenvolvimento da floresta. A estimação do crescimento é uma etapa essencial no ordenamento florestal. Qualquer planejamento implica na predição do crescimento (SPURR, 1952).

ALDER (1980), CLUTTER et allii (1983) e DAVIS e JOHNSON (1987) classificaram os modelos de crescimento e produção em três tipos:

- modelos globais do povoamento, que permitem obter uma estimativa geral da produção por unidade de área;

- modelos por classe diamétrica, que possibilitam a prognose do número de árvores por classe de diâmetro. A altura, o volume e outras características do povoamento podem ser associadas a cada uma dessas classes; $\mathrm{e}$,

- modelos para árvores individuais, que consideram características de árvores individuais para a prognose do crescimento e produção do povoamento.

Segundo SANQUETTA (1996), três modelos não espaciais expressam o desenvolvimento do povoamento através da descrição da evolução das distribuições diamétricas ou de outra variável em classes, e são conhecidos como funções probabilísticas, matrizes de transição e processos de difusão.

A matriz de transição é um processo estocástico utilizado para estudar fenômenos que passam, a partir de um estado inicial, por uma seqüência de 
estados, onde a transição de um determinado estado ocorre segundo uma certa probabilidade. Os pontos mais importantes na montagem de uma Cadeia de Markov são a definição de estados do sistema e a construção da matriz de transição probabilística (HOYOS, 1980). De acordo com ENRIGHT e OGDEN (1979), o único requisito para a utilização do modelo matricial é que a população possa ser dividida em estados ou compartimentos, e que haja a probabilidade de movimento de um estado para outro no tempo.

De acordo com SANQUETTA (1996), nas matrizes de transição utilizase o critério de separar árvores de uma certa classe diamétrica que crescem para uma, duas ou mais classes consecutivas daquelas que permanecem na mesma classe ou morrem durante um intervalo de tempo. Essa dinâmica nas classes determina as probabilidades que constituem os elementos da matriz de transição.

A projeção do crescimento diamétrico com a utilização da matriz de transição já foi estudada por diversos pesquisadores em várias partes do mundo. Entre eles, pode-se citar BRUNER e MOSER JR. (1973) em um povoamento de folhosas mistas em Wisconsin-EUA, ENRIGHT e OGDEN (1979) em populações de Araucaria sp. na floresta tropical úmida de Papua-Nova Guiné e de Nothofagus fusca nas florestas temperadas da Nova Zelândia, BUONGIORNO e MICHIE (1980) em povoamentos de Acer sacharum em Wisconsin e Michigan nos EUA, ROBERT e HRUSKA (1986) em povoamentos de Pinus sp. nos Estados Unidos, e MENDOZA e SETYARSO (1986) como instrumento auxiliar na determinação do ciclo de corte em florestas da Indonésia.

LOWELL e MITCHELL (1987) apresentaram três importantes limitações da Cadeia de Markov: 1) uma única qualidade de sítio é assumida enquanto a dinâmica da população varia com o sítio; 2) qualquer árvore é tratada da mesma maneira independentemente das características particulares do povoamento; 3) a estrutura do povoamento é ignorada no sentido da dinâmica da população ser considerada a mesma, quer seja o povoamento coetâneo ou dissetâneo, quer o povoamento seja puro ou tenha uma composição de espécies.

Um grande inconveniente da utilização da Cadeia de Markov é a inflexibilidade do modelo em não fazer projeções sobre intervalos que não sejam múltiplos do intervalo de medição. Neste caso, o estabelecimento de estratégias de manejo fica também condicionado aos anos múltiplos do intervalo de medição (HARRISON e MICHIE, 1985). Estes autores desenvolveram um procedimento para contornar esta restrição, através da fatorização da matriz de transição para períodos de um ano.

O objetivo principal do presente estudo é avaliar e simular o crescimento de povoamentos jovens, coetâneos, monoespecíficos e homogêneos de Pinus taeda utilizando a técnica de simulação Matrizes de Transição.

\section{MATERIAL E MÉTODOS}

Os dados utilizados são provenientes de um experimento de espaçamento em Pinus taeda L., instalado no município de Jaguariaíva - PR, na Fazenda 
Lageado, de propriedade da "Pisa Florestal", Projeto J-2, talhão 11. A implantação ocorreu entre 28 de outubro e 09 de novembro de 1987, com mudas obtidas a partir de sementes procedentes de Telêmaco Borba - PR, da empresa "Klabin Florestal".

A área ocupada pelo experimento consta de seis blocos instalados de maneira contínua em um total de 2,97 ha (incluindo a bordadura), com uma área útil de 2,59 ha. O local de implantação do experimento possuía reflorestamento com Pinus taeda com 16 anos de idade. No final de 1986, foi executado um corte raso no projeto. A prática de limpeza do material resultante da exploração foi uma queima controlada, ficando a área segundo os critérios de preparo de terreno da empresa, apta a receber nova implantação.

$\mathrm{O}$ experimento avalia cinco diferentes intensidades de espaçamentos, repetidos em seis blocos contínuos. Cada parcela possui bordadura. Além disto, o teste possui uma bordadura dupla externa procurando proporcionar o máximo de confiabilidade às inferências.

Os tratamentos do Delineamento em Blocos ao Acaso são os seguintes:

\begin{tabular}{cccccc}
\hline Tratamento & $\begin{array}{c}\text { Espaçamento } \\
(\mathrm{m})\end{array}$ & Árv./ha & \multicolumn{2}{c}{ Área da Parcela $\left(\mathrm{m}^{2}\right)$} & Plantas \\
\cline { 4 - 5 } & & & total & útil & úteis \\
\hline 1 & $2,5 \times 1,2$ & 3333 & 630 & 234 & 78 \\
2 & $2,5 \times 2,0$ & 2000 & 630 & 210 & 42 \\
3 & $2,5 \times 2,8$ & 1428 & 810 & 315 & 45 \\
4 & $2,5 \times 3,6$ & 1111 & 990 & 378 & 42 \\
5 & $2,5 \times 4,4$ & 909 & 1260 & 440 & 40 \\
\hline
\end{tabular}

Foram inicialmente mensuradas somente as alturas das árvores úteis das parcelas. Após as árvores atingirem altura superior a 1,3 m, as medições foram efetuadas obtendo-se os valores de CAP e altura total. As medições de CAP e da altura total foram realizadas anualmente, entre os anos 1991 e 1996. A estrutura e a confiabilidade das medições realizadas permitiu utilizar os dados de árvores individuais provenientes das medições realizadas em 1993 e 1996 ( $6^{\circ}$ e $9^{\circ}$ anos), devido a que são as medições confiáveis mais distantes entre si no tempo.

A primeira análise a ser realizada sobre os dados será a de testar a existência de diferenças entre os 5 tratamentos que justifiquem o estudo separado do crescimento de cada um deles. As diferenças serão analisadas através do teste de comparação de médias (Tukey, $\alpha=0,05$ ), para os 5 tratamentos e os 6 períodos de observação (1991 a 1996).

A matriz de transição é um método de simulação que considera as frequiências diamétricas no ano de início da simulação $\left(N_{t}\right)$ na forma de vetor, que multiplicado por uma matriz de probabilidades de transição entre classes diamétricas $(T p)$ permite obter as freqüências diamétricas do ano final $\left(N_{t+1}\right)$. O recrutamento é considerado também como um vetor $\left(R_{t}\right)$, que é adicionado ao produto vetor-matriz descrito. A mortalidade pode ser considerada de duas maneiras: como um vetor de subtração $(M t)$, ou incluída na matriz $T p$ nas últimas linha e coluna. 
Em álgebra de matrizes, o processo de simulação para 1 período $(t \rightarrow$ $t+1$ ), com a mortalidade incluída na matriz de transição, pode ser resumido como segue:

$$
\underset{(n+1) \times 1}{N_{\mathfrak{t}+1}}=\left(\underset{t \times(n+1)}{N_{t}^{\prime}} \cdot \underset{(n+1) \times(n+1)}{T} \mathfrak{T}^{\prime} \quad\right)+\underset{(n+1) \times 1}{R_{t}}
$$

e para simulações abrangendo $p$ períodos sem considerar o recrutamento, que é o caso das florestas plantadas de Pinus sp., o processo de simulação fica:

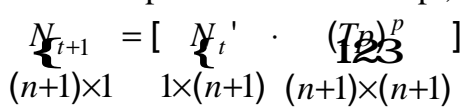

Onde $(T p)^{p}$ é a matriz potência de $T p$, o que em álgebra de matrizes indica o produto da matriz $T p$ por si mesma $p$ vezes, i.e. $T p^{3}=(T p \times T p \times T p)$. Para que o produto seja factível, a matriz $T p$ deve ser quadrada ( $\mathrm{N}^{\circ}$ de linhas $=\mathrm{N}^{\circ}$ de colunas).

Os vetores de frequiências observadas $\left(N_{t}\right)$ e de recrutamento $\left(R_{t}\right)$ representam unidades físicas (árvores) por classes de diâmetro presentes no início da simulação e recrutadas durante o período $t+1$, respectivamente. Por se tratar de florestas plantadas o recrutamento pode ser desprezado, e o vetor $R_{t}$ considerado como um vetor nulo. O vetor $N_{t}$ é representado como segue:

$$
N_{t}^{\prime}=\left[\begin{array}{lllllll}
N_{1} & N_{2} & N_{3} & \mathrm{~L} & N_{n-1} & N_{n} & N_{m}
\end{array}\right]^{\prime}
$$

onde: $\quad N_{i}=$ freqüência diamétrica da classe $i$

$i=1,2, \ldots$,

$\mathrm{n}-1, \mathrm{n} ; \quad N_{m}=$ número de árvores mortas no instante $t$.

A matriz de transição (Tp) essencialmente é uma matriz de probabilidades, cujos elementos estão descritos a seguir:

$$
T p=\left[\begin{array}{cccccccccc}
p f_{1} & p a_{1} & p b_{1} & p c_{1} & \mathrm{~L} & \mathrm{~L} & \mathrm{~L} & \mathrm{~L} & \mathrm{~L} & p m_{1} \\
& p f_{2} & p a_{2} & p b_{2} & p c_{2} & \mathrm{~L} & \mathrm{~L} & \mathrm{~L} & \mathrm{~L} & p m_{2} \\
& & p f_{3} & p a_{3} & p b_{3} & p c_{3} & \mathrm{~L} & \mathrm{~L} & \mathrm{~L} & p m_{3} \\
& & & & \mathrm{M} & \mathrm{M} & & & & \mathrm{M} \\
& & & & p f_{i} & p a_{i} & p b_{i} & p c_{i} & & p m_{i} \\
& & & & & & & \mathrm{M} & \mathrm{M} & \mathrm{M} \\
& & & & & & & \mathrm{M} & \mathrm{M} & \mathrm{M} \\
& & & & & & & p f_{n-1} & p a_{n-1} & p m_{n-1} \\
& & & & & & & & p f_{n} & p m_{n} \\
& & & & & & & & & 1
\end{array}\right]
$$


onde:

$p f_{i}=$ probabilidade das árvores da classe $i$ ficarem na classe $i$ durante $(t \rightarrow t+1)$; $p a_{i}=$ probabilidade das árvores da classe $i$ avançarem para a classe $i+1(t \rightarrow t+1)$; $p b_{i}=$ probabilidade das árvores da classe $i$ avançarem para a classe $i+2(t \rightarrow t+1)$; $p c_{i}=$ probabilidade das árvores da classe $i$ avançarem para a classe $i+3(t \rightarrow t+1)$; $\mathrm{pm}_{i}=$ probabilidade das árvores da classe $i$ morrerem durante o período $(t \rightarrow t+1)$; $i=$ número da classe diamétrica, variando desde 1 até $n$ (última classe).

Com os dados obtidos nos anos 1993 e 1996 será determinado, para cada tratamento e classe diamétrica, o número de árvores que permaneceram, morreram ou passaram para a(s) classe(s) seguinte(s), durante o período considerado. Com estes valores poderão ser calculadas as probabilidades de permanência na classe, transição entre classes e mortalidade das árvores de cada tratamento e classe diamétrica, necessárias para a construção das matrizes de transição.

A mortalidade será incluída na matriz de transição nas últimas linha e coluna. Por se tratar de um reflorestamento medido a partir do $4^{\underline{0}}$ ano com o replantio já realizado, o recrutamento será considerado inexistente.

Serão obtidas matrizes para classes diamétricas de diferentes amplitudes, considerando as diferentes densidades dos tratamentos. A escolha da amplitude de classe para cada tratamento dependerá da aparência da matriz de transição resultante e, principalmente, do resultado das simulações realizadas.

\section{RESULTADOS E DISCUSSÃO}

O teste de comparação de médias de diâmetros por tratamentos mostra que a partir do ano 1992 começam a ser observadas diferenças significativas $(\alpha<0,05)$ entre alguns tratamentos. As diferenças tornam-se mais notáveis ao longo do tempo, até distinguir quase todos os tratamentos entre si no ano 1996. A Figura 1 mostra os resultados obtidos no teste de comparação de médias de diâmetros.

Até o 9ํano de idade (1996), as médias dos diâmetros dos tratamentos $3 \mathrm{e}$ 4 (1428 e 1111 árv./ha respectivamente) não apresentaram diferenças significativas entre si através do Teste de Tukey ao nível de significância de 5\%. Todos os demais tratamentos estão claramente diferenciados. Considerando que a avaliação do ensaio de espaçamentos ao $9^{\underline{0}}$ ano para Pinus taeda é relativamente precoce, é prudente, para os efeitos desta avaliação e simulação do crescimento, discriminar os 5 tratamentos. Entretanto um erro maior seria cometido se os tratamentos $3 \mathrm{e} 4$ fossem analisados de maneira agrupada e, hipoteticamente com o tempo, se diferenciassem, do que se os tratamentos fossem analisados separadamente e, também em hipótese, com o tempo não se diferenciassem. A opção de agrupar os tratamentos em anos subseqüentes sempre é factível.

Também foram comparadas as médias entre as 6 repetições (blocos), não apresentando diferenças significativas no Teste de comparação de médias de Tukey ao nível de significância de 5\%. 
Figura 1 - Análise de amplitude múltipla (Tukey, $\alpha=0,05)$ para a comparação de médias de diâmetros por tratamento. Anos 1991 a 1996

Figure 1 -Multiple range analysis (Tukey $\alpha=0,05)$ for treatment means diameter comparation. Years 1991 to 1996

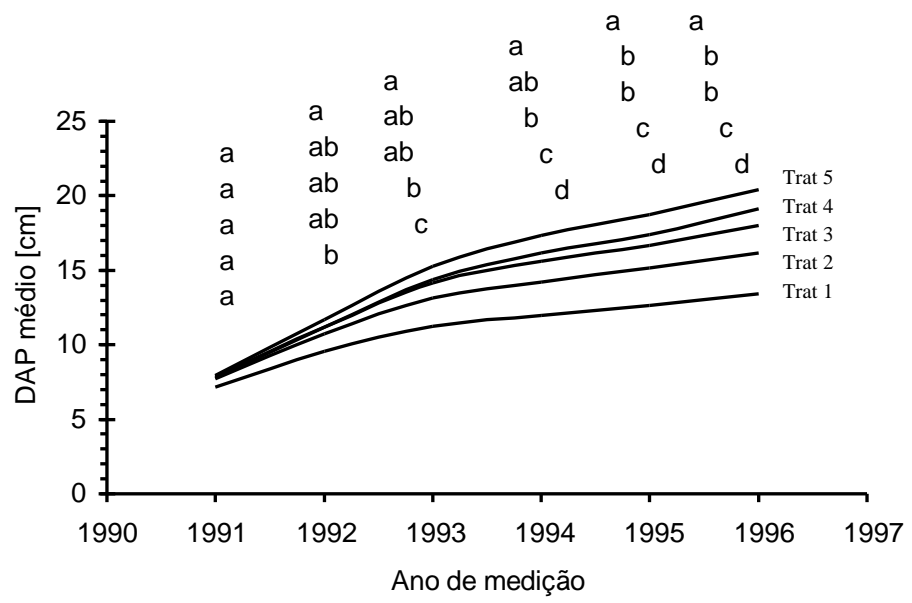

Por terem sido avaliadas densidades desde 900 até 3333 plantas por hectare, foi necessário testar amplitudes de classe diamétrica diferentes, de 2, 3 e 4 $\mathrm{cm}$. Esta decisão baseou-se no fato de que, ao utilizar classes diamétricas de pequena amplitude para espaçamentos amplos (densidades baixas), as probabilidades das árvores ficarem na mesma classe $\left(p f_{i}\right)$ diminuíram e inclusive chegaram a se anular, enquanto que as probabilidades das árvores avançarem 2,3 , 4 e até 5 classes $\left(p b_{i}, p c_{i}, p d_{i}, p e_{i}\right)$ aumentaram. Isto conduz a uma matriz de transição imprópria, de difícil interpretação, onde a diagonal principal possui elementos quase todos nulos $\left(p f_{i}=0\right)$. Por outro lado, quando foram utilizadas amplitudes de classes diamétricas grandes para espaçamentos pequenos (densidades altas), as $p f_{i}$ aumentaram enquanto que as $p a_{i}, p b_{i}$ e $p c_{i}$ diminuíram.

Foram construídas matrizes para um período de três anos, considerando os dados coletados em 1993 e 1996. Para a construção das matrizes foi elaborada uma rotina esquematizada no fluxograma em anexo. A rotina gerou as cinco matrizes de transição associadas aos cinco tratamentos, além dos vetores de frequiências diamétricas observadas para os anos de 1993, 1996 e simulados para o ano de 1999. Para cada tratamento foram geradas matrizes com amplitudes de classes diamétricas de 2, $3 \mathrm{e} 4 \mathrm{~cm}$, resultando em um total 15 matrizes de transição.

As distribuições diamétricas observadas nos anos 1993 e 1996, e simuladas para o ano 1999, são apresentadas na Figura 2 para os 5 tratamentos. A Figura 2 mostra uma harmonia própria da dinâmica de um processo biológico, onde as medidas de posição (média, moda) aumentam com o tempo, junto com o 
aumento das medidas de dispersão (Figs. 2c e 2e). As pequenas discrepâncias observadas nas Figuras $2 \mathrm{a}, 2 \mathrm{~b}$ e $2 \mathrm{~d}$ podem ser resultantes do fato de se estar trabalhando com amostras pequenas e povoamentos jovens, não se tendo informação do ciclo completo da dinâmica da floresta.

Figura 2 - Distribuições diamétricas observadas $(1993 ; 1996)$ e simuladas (1999) para os tratamentos 1 (a), 2 (b), 3 (c), 4 (d) e 5 (e)

Figure 2 - Observed (1993; 1996) and simulated (1999) diameter distributions for treatments $1(a), 2(b), 3(c), 4(d)$ and $5(e)$

a)

Tratamento 1 - Classes de DAP de $2 \mathrm{~cm}$.

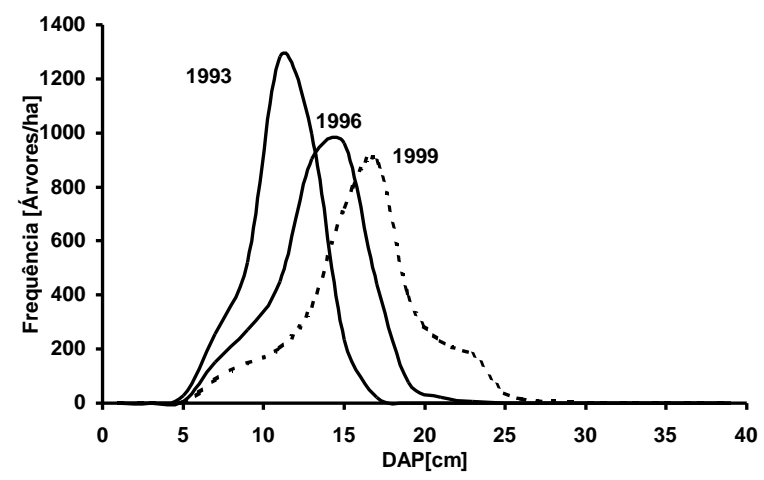

b)

Tratamento 2 - Classes de DAP de $3 \mathrm{~cm}$.

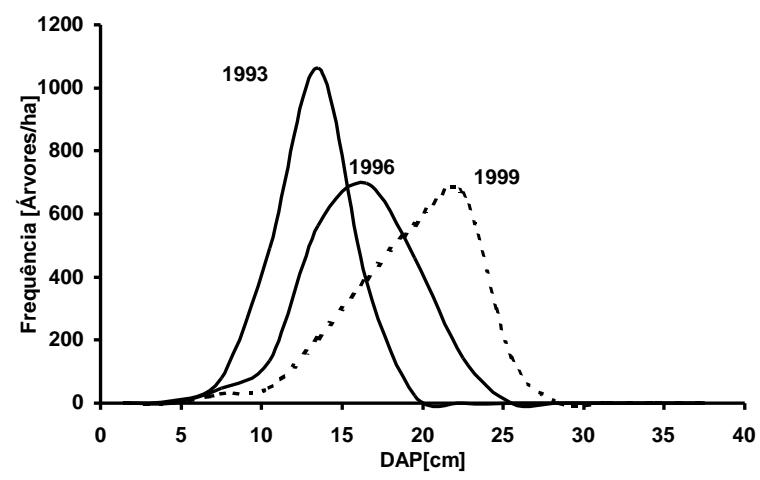


c)

Tratamento 3 - Classesde DAP de $3 \mathrm{~cm}$.

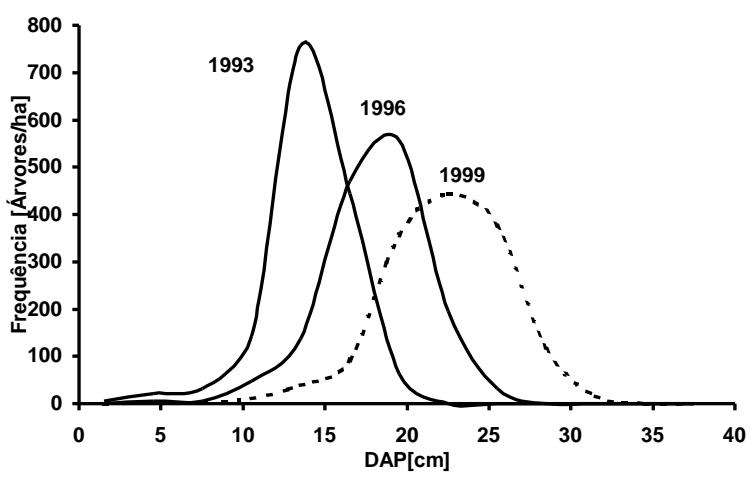

d)

Tratamento 4 - Classes de DAP de $3 \mathrm{~cm}$.

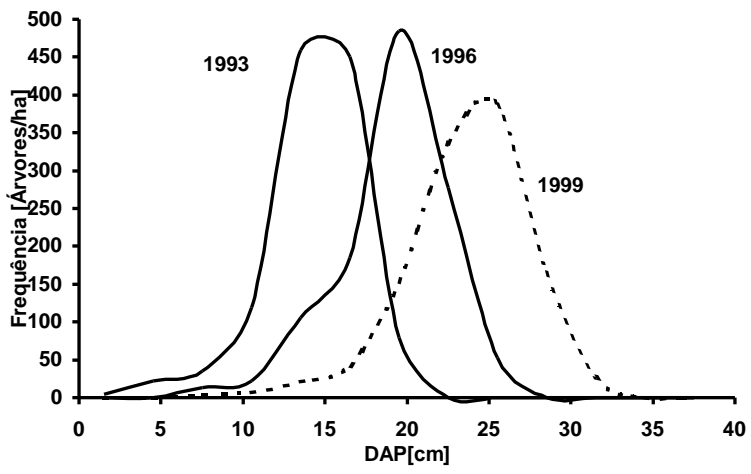


e)

Tratamento 5 - Classes de DAP de $4 \mathrm{~cm}$.

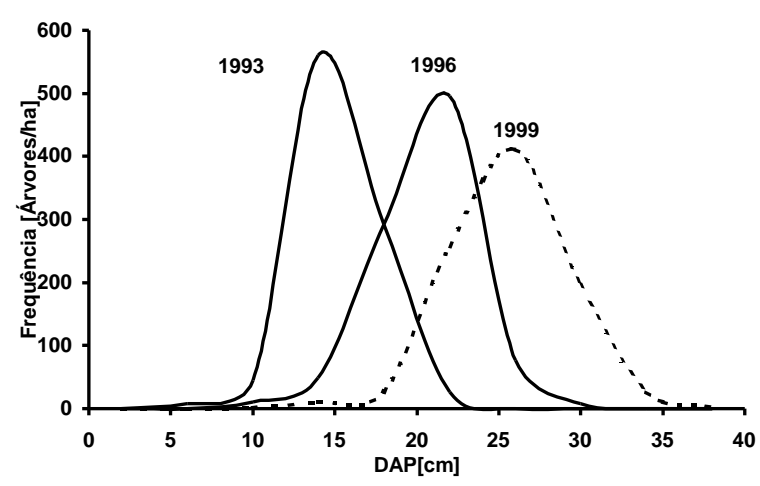

As matrizes escolhidas para os 5 tratamentos são apresentadas nas Tabelas 1, 2, 3, 4 e 5 . 
Tabela1 - Matriz de transição obtida para o tratamento 1 com classes diamétricas de $2 \mathrm{~cm}$ Table 1 - Transition matrix obtained for treatment 1 with $2 \mathrm{~cm}$ diameter classes

\begin{tabular}{|c|c|c|c|c|c|c|c|c|c|c|c|c|c|c|c|c|c|c|c|c|c|}
\hline 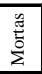 & 0 & 0 & $\begin{array}{l}0 \\
n \\
0\end{array}$ & $\stackrel{\infty}{\circ}$ & $\tilde{o}_{0}$ & 0 & 0 & 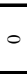 & 0 & 0 & 0 & 0 & 0 & 0 & 0 & 0 & 0 & 0 & 0 & 0 & $\stackrel{8}{-}$ \\
\hline 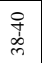 & ' & ' & ' & . & ' & ' & ' & & & 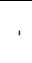 & & ' & ' & ' & ' & & $\hat{\tilde{0}}$ & & 8 & $\underset{-}{8}$ & \\
\hline 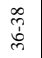 & . & . & . & . & . & ' & . & ' & 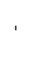 & I & 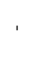 & . & . & . & ' & $\tilde{m}_{0}$ & $\overbrace{0}^{2}$ & $m_{0}^{3}$ & 0 & , & \\
\hline 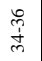 & . & . & . & . & ' & ' & . & ' & 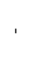 & ' & 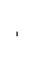 & ' & . & . & $\mathrm{m}_{0}^{m}$ & $m_{0}^{m}$ & $m_{0}^{2}$ & 0 & ' & , & \\
\hline $\begin{array}{l}\text { d. } \\
\text { dे }\end{array}$ & . & . & . & , & . & ' & ' & 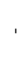 & 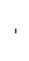 & ' & & ' & ' & $\tilde{3}_{0}$ & $m_{0}^{m}$ & $\tilde{m}_{0}^{2}$ & o & ' & ' & ' & \\
\hline స్లి & . & , & ' & . & . & . & . & . & r & ' & ' & . & के & हु. & $\overbrace{0}^{m}$ & 0 & ' & ' & ' & ' & , \\
\hline $\begin{array}{l}\vec{D} \\
\dot{d} \\
\dot{d}\end{array}$ & ' & . & . & . & . & ' & ' & ' & & ' & & $\hat{m}_{0}$ & $\hat{m}_{0}^{2}$ & $\tilde{m}_{0}^{2}$ & 0 & ' & ' & ' & ' & ' & \\
\hline 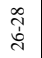 & . & . & ' & . & ' & ' & ' & ' & & ' & $\tilde{m}_{0}$ & $\hat{m}_{0}$ & लై & 0 & ' & ' & ' & . & ' & ' & \\
\hline 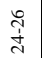 & . & . & . & . & , & ' & . & 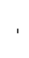 & & $\mathrm{m}_{0}$ & $m_{0}^{m}$ & $\hat{m}_{0}$ & 0 & , & , & 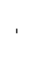 & ' & ' & ' & ' & \\
\hline 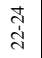 & . & . & . & . & . & ' & , & ' & $m$ & ले & $m_{0}^{3}$ & 0 & , & ' & ' & ' & ' & ' & ' & ' & 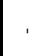 \\
\hline $\begin{array}{l}\text { สิ } \\
\text { ते }\end{array}$ & . & , & . & . & . & , & . & 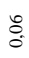 & 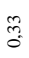 & $\mathrm{m}_{0}$ & 0 & . & ' & ' & ' & ' & ' & , & ' & , & . \\
\hline 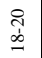 & . & . & . & . & . & . & $\bar{o}_{0}$ & I & ? & 0 & . & ' & ' & ' & ' & ' & ' & ' & ' & ' & 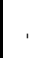 \\
\hline$\frac{\infty}{\underline{b}}$ & . & , & . & . & . & $\overrightarrow{0}_{0}$ & $\begin{array}{l}\text { : } \\
0\end{array}$ & $\stackrel{5}{\circ}$ & 0 & ' & . & ' & , & , & , & 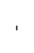 & , & . & ' & , & ' \\
\hline$\stackrel{0}{ \pm}$ & ' & ' & . & . & 0 & נै & : & ${ }_{0}^{\circ}$ & ' & ' & ' & ' & ' & ' & ' & ' & . & ' & ' & ' & . \\
\hline$\frac{ \pm}{\dot{I}}$ & . & ' & , & 0 & $\frac{7}{0}$ & $\stackrel{\vec{b}}{\circ}$ & $\stackrel{\circ}{\circ}$ & 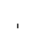 & 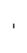 & ' & ' & ' & ' & ' & ' & . & ' & ' & ' & ' & \\
\hline$\frac{\mathfrak{T}}{\varrho}$ & . & . & 0 & 0 & $\bar{n}$ & $\frac{ \pm}{0}$ & ' & ' & ' & . & . & ' & , & , & , & ' & , & ' & ' & , & ' \\
\hline$\frac{\circ}{\infty}$ & ' & 0 & 0 & $\overbrace{0}^{\infty}$ & ${ }_{0}^{+}$ & ' & ' & 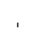 & ' & . & . & ' & ' & ' & ' & ' & . & . & ' & ' & , \\
\hline $\begin{array}{l}\infty \\
\dot{b}\end{array}$ & 0 & 0 & तू & : & ' & ' & . & ' & ' & . & ' & ' & ' & ' & ' & ' & , & ' & ' & , & . \\
\hline$\stackrel{0}{+}$ & 0 & 0 & तू & . & ' & , & . & ' & ' & , & . & ' & ' & ' & ' & ' & ' & ' & ' & ' & , \\
\hline$\stackrel{J}{\sim}$ & 0 & $\stackrel{8}{-}$ & ' & ' & ' & ' & ' & ' & ' & ' & ' & ' & ' & ' & , & ' & , & ' & ' & , & ' \\
\hline \multirow[t]{2}{*}{ 定 } & $\underset{-}{\stackrel{-}{-}}$ & ' & ' & . & ' & , & . & & & . & & . & . & ' & , & . & . & . & ' & , & , \\
\hline & $\stackrel{\partial}{\partial}$ & $\stackrel{\Delta}{\sim}$ & $\stackrel{0}{+}$ & $\begin{array}{l}\infty \\
0 \\
b\end{array}$ & $\frac{\rho}{\infty}$ & $\frac{7}{0}$ & $\stackrel{ \pm}{\mathrm{I}}$ & & & 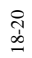 & ?2 & 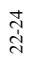 & $\underset{I}{\mathbb{T}}$ & $\begin{array}{l}\text { \%े } \\
\text { ک్ర }\end{array}$ & 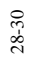 & స్తి & ते & 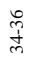 & 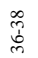 & $\begin{array}{l}\substack{q \\
\infty \\
\infty} \\
\text { d. }\end{array}$ & 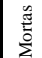 \\
\hline
\end{tabular}


Tabela 2 - Matriz de transição obtida para o tratamento 2 com classes diamétricas de $3 \mathrm{~cm}$

Table 2 - Transition matrix obtained for treatment 2 with $3 \mathrm{~cm}$ diameter classes

\begin{tabular}{|c|ccccccccccccc|c|}
\hline & $>0-3$ & $3-6$ & $6-9$ & $9-12$ & $12-15$ & $15-18$ & $18-21$ & $21-24$ & $24-27$ & $27-30$ & $30-33$ & $33-36$ & $36-39$ & Mortas \\
\hline$>0-3$ & 1,00 & 0 & 0 & 0 & - & - & - & - & - & - & - & - & - & 0 \\
$3-6$ & - & 0 & 0 & 0 & 0 & - & - & - & - & - & - & - & - & 1,00 \\
$6-9$ & - & - & 0,60 & 0,40 & 0 & 0 & - & - & - & - & - & - & - & 0 \\
$9-12$ & - & - & - & 0,21 & 0,74 & 0,05 & 0 & - & - & - & - & - & - & 0 \\
$12-15$ & - & - & - & - & 0,19 & 0,61 & 0,19 & 0,01 & - & - & - & - & - & 0 \\
$15-18$ & - & - & - & - & - & 0,06 & 0,65 & 0,29 & 0 & - & - & - & - & 0 \\
$18-21$ & - & - & - & - & - & - & 0 & 1,00 & 0 & 0 & - & - & - & 0 \\
$21-24$ & - & - & - & - & - & - & - & 0 & 1,00 & 0 & 0 & - & - & 0 \\
$24-27$ & - & - & - & - & - & - & - & - & 0 & 1,00 & 0 & 0 & - & 0 \\
$27-30$ & - & - & - & - & - & - & - & - & - & 0 & 1,00 & 0 & 0 & 0 \\
$30-33$ & - & - & - & - & - & - & - & - & - & - & 0 & 1,00 & 0 & 0 \\
$33-36$ & - & - & - & - & - & - & - & - & - & - & - & 0 & 1,00 & 0 \\
$36-39$ & - & - & - & - & - & - & - & - & - & - & - & - & 1,00 & 0 \\
\hline Mortas & - & - & - & - & - & - & - & - & - & - & - & - & - & 1,00 \\
\hline
\end{tabular}

Tabela 3 - Matriz de transição obtida para o Tratamento 3 a partir de classes diamétricas de $3 \mathrm{~cm}$.

Table 3 - Transition matrix obtained for treatment 3 with $3 \mathrm{~cm}$ diameter classes.

\begin{tabular}{|c|ccccccccccccc|c|}
\hline & $>0-3$ & $3-6$ & $6-9$ & $9-12$ & $12-15$ & $15-18$ & $18-21$ & $21-24$ & $24-27$ & $27-30$ & $30-33$ & $33-36$ & $36-39$ & Mortas \\
\hline$>0-3$ & 0 & 0 & 0 & 0 & - & - & - & - & - & - & - & - & - & 1,00 \\
$3-6$ & - & 0,25 & 0,25 & 0 & 0 & - & - & - & - & - & - & - & - & 0,50 \\
$6-9$ & - & - & 0 & 1,00 & 0 & 0 & - & - & - & - & - & - & - & 0 \\
$9-12$ & - & - & - & 0,11 & 0,59 & 0,30 & 0 & - & - & - & - & - & - & 0 \\
$12-15$ & - & - & - & - & 0,07 & 0,57 & 0,35 & 0,01 & - & - & - & - & - & 0 \\
$15-18$ & - & - & - & - & - & 0 & 0,65 & 0,35 & 0 & - & - & - & - & 0 \\
$18-21$ & - & - & - & - & - & - & 0 & 0,50 & 0,50 & 0 & - & - & - & 0 \\
$21-24$ & - & - & - & - & - & - & - & 0 & 0,50 & 0,50 & 0 & - & - & 0 \\
$24-27$ & - & - & - & - & - & - & - & - & 0 & 0,50 & 0,50 & 0 & - & 0 \\
$27-30$ & - & - & - & - & - & - & - & - & - & 0 & 0,50 & 0,50 & 0 & 0 \\
$30-33$ & - & - & - & - & - & - & - & - & - & - & 0 & 0,50 & 0,50 & 0 \\
$33-36$ & - & - & - & - & - & - & - & - & - & - & - & 0 & 1,00 & 0 \\
$36-39$ & - & - & - & - & - & - & - & - & - & - & - & - & 1,00 & 0 \\
\hline Mortas & - & - & - & - & - & - & - & - & - & - & - & - & - & 1,00 \\
\hline
\end{tabular}


Tabela 4 - Matriz de transição obtida para o Tratamento 4 a partir de classes diamétricas de $3 \mathrm{~cm}$

Table 4 - Transition matrix obtained for treatment 4 with $4 \mathrm{~cm}$ diameter classes

\begin{tabular}{|c|ccccccccccccc|c|}
\hline & $>0-3$ & $3-6$ & $6-9$ & $9-12$ & $12-15$ & $15-18$ & $18-21$ & $21-24$ & $24-27$ & $27-30$ & $30-33$ & $33-36$ & $36-39$ & Mortas \\
\hline$>0-3$ & 0 & 0 & 0 & 0 & - & - & - & - & - & - & - & - & - & 1,00 \\
$3-6$ & - & 0 & 0,20 & 0 & 0 & - & - & - & - & - & - & - & - & 0,80 \\
$6-9$ & - & - & 0,25 & 0,50 & 0,13 & 0 & - & - & - & - & - & - & - & 0,13 \\
$9-12$ & - & - & - & 0,04 & 0,67 & 0,30 & 0 & - & - & - & - & - & - & 0 \\
$12-15$ & - & - & - & - & 0,05 & 0,32 & 0,58 & 0,06 & - & - & - & - & - & 0 \\
$15-18$ & - & - & - & - & - & 0,01 & 0,49 & 0,47 & 0,03 & - & - & - & - & 0 \\
$18-21$ & - & - & - & - & - & - & 0 & 0,50 & 0,50 & 0 & - & - & - & 0 \\
$21-24$ & - & - & - & - & - & - & - & 0 & 0,50 & 0,50 & 0 & - & - & 0 \\
$24-27$ & - & - & - & - & - & - & - & - & 0 & 0,50 & 0,50 & 0 & - & 0 \\
$27-30$ & - & - & - & - & - & - & - & - & - & 0 & 0,50 & 0,50 & 0 & 0 \\
$30-33$ & - & - & - & - & - & - & - & - & - & - & 0 & 0,50 & 0,50 & 0 \\
$33-36$ & - & - & - & - & - & - & - & - & - & - & - & 0 & 1,00 & 0 \\
$36-39$ & - & - & - & - & - & - & - & - & - & - & - & - & 1,00 & 0 \\
\hline Mortas & - & - & - & - & - & - & - & - & - & - & - & - & - & 1,00 \\
\hline
\end{tabular}

Tabela 5 - Matriz de transição obtida para o Tratamento 5 a partir de classes diamétricas de $4 \mathrm{~cm}$

Table 5 - Transition matrix obtained for treatment 5 with $4 \mathrm{~cm}$ diameter classes

\begin{tabular}{|c|ccccccccccc|c|}
\hline & $>0-4$ & $4-8$ & $8-12$ & $12-16$ & $16-20$ & $20-24$ & $24-28$ & $28-32$ & $32-36$ & $36-40$ & Mortas \\
\hline$>0-4$ & 1,00 & 0 & 0 & 0 & - & - & - & - & - & - & 0 \\
$4-8$ & - & 0 & 1,00 & 0 & 0 & - & - & - & - & - & 0 \\
$8-12$ & - & - & 0,08 & 0,75 & 0,17 & 0 & - & - & - & - & 0 \\
$12-16$ & - & - & - & 0,03 & 0,51 & 0,47 & 0 & - & - & - & 0 \\
$16-20$ & - & - & - & - & 0 & 0,81 & 0,19 & 0 & - & - & 0 \\
$20-24$ & - & - & - & - & - & 0 & 0,71 & 0,29 & 0 & - & 0 \\
$24-28$ & - & - & - & - & - & - & 0 & 0,71 & 0,29 & 0 & 0 \\
$28-32$ & - & - & - & - & - & - & - & 0 & 0,71 & 0,29 & 0 \\
$32-36$ & - & - & - & - & - & - & - & - & 0 & 1,00 & 0 \\
$36-40$ & - & - & - & - & - & - & - & - & - & 1,00 & 0 \\
\hline Mortas & - & - & - & - & - & - & - & - & - & - & 1,00 \\
\hline
\end{tabular}

Nas matrizes de transição apresentadas nas Tabelas 1 a 5 podem ser observadas algumas anomalias, uma vez que as probabilidades $p f_{1}$ e $p f_{2}$ da matriz de transição do tratamento 1 têm valor 1 (100\%) (Tabela 1). Isto quer dizer que todas as árvores destas classes diamétricas permanecerão em suas respectivas classes, sem existir transição para as classes seguintes. Já na matriz de transição do tratamento 2, o valor da probabilidade das árvores da classe diamétrica 2 morrerem $\left(\mathrm{pm}_{2}\right)$ tem valor $1(100 \%)$, dizendo que todas as árvores desta classe diamétrica, morrerão no seguinte período de simulação de três anos (Tabela 2). Esta observação pode ser feita para as $p m_{l}$ das matrizes de transição dos tratamentos 3 e 4 (Tabelas 3 e 4).

Devido à precocidade da avaliação, as probabilidades de transição disponíveis a partir dos dados observados correspondem somente às primeiras classes diamétricas. No tratamento 1, as probabilidades de transição foram observadas até a classe diamétrica de $16-18 \mathrm{~cm}$, nos tratamentos 2,3 e 4 , até a 
classe diamétrica de 18-21 cm, e no tratamento 5 até a classe diamétrica de 20-24 $\mathrm{cm}$. Em conseqüência foi necessário assumir que as últimas probabilidades de transição observadas para os 5 tratamentos se mantêm constantes para todas as classes subseqüentes. Desta maneira é satisfeito o requisito de que a somatória das probabilidades de todas as linhas da matriz de transição deve ser igual a 1. Caso contrário, se "perderiam” árvores durante a simulação.

\section{CONCLUSÕES E RECOMENDAÇÕES}

A construção de matrizes de transição a partir de observações diamétricas obtidas de povoamentos jovens, coetâneos, monoespecíficos e homogêneos de Pinus taeda em duas idades é factível e permite obter simulações coerentes para um período igual ao intervalo entre estas idades. No caso analisado, as observações foram realizadas no $6^{0}$ e $9^{0}$ anos (1993 e 1996), e permitiram simular as freqüências diamétricas para o $12^{\circ}$ ano (1999). As simulações obtidos mostram uma harmonia própria da dinâmica de um processo biológico, onde as medidas de posição (média, moda) aumentam com o tempo, junto com o aumento das medidas de dispersão.

Já a simulação para intervalos de tempo maiores do que o período considerado para a construção das matrizes de transição é desaconselhável, por se tratar de povoamentos jovens e não estar disponível, portanto, informação do ciclo completo da dinâmica da floresta.

Um aspecto questionável da simulação com matrizes de transição é a suposição de que as probabilidades, uma vez calculadas e inseridas na matriz de transição, permanecerão constantes ao longo de toda a vida da floresta. Seria recomendável prosseguir com as observações e medições da floresta até o final do ciclo, tanto para validar os resultados obtidos com as simulações apresentadas neste estudo, quanto para calibrar e construir matrizes de transição para períodos e idades diferentes, de maneira que a simulação das distribuições diamétricas se torne dinâmica, utilizando sucessivamente várias matrizes de transição até atingir o final do ciclo.

Finalmente, é necessário recomendar que estes estudos comecem a ser realizados com dados provenientes de amostras de povoamentos não experimentais, para serem contrastados os resultados com aqueles obtidos a partir dos ensaios. É requisito fundamental de qualquer modelo de simulação florestal, a constante calibragem e re-alimentação com dados provenientes de povoamentos comerciais.

\section{AGRADECIMENTOS}

Os autores desejam agradecer de maneira muito especial à empresa Pisa Florestal por ter facilitado gentilmente os dados utilizados neste trabalho, em especial ao Engenheiro Romualdo Maestri, pioneiro deste experimento. 
Participaram deste trabalho, como colaboradores, as seguintes pessoas: Fernando dos S. Gomes, Zenóbio A. G. P. da Gama e Silva, Ruth E. Loch, Eduardo C. da Cruz e Alexandra C. P. S. Barcoszeck.

\section{BIBLIOGRAFIA CITADA}

ALDER, D., 1980. Estimación del volumen forestal y predicción del rendimiento con especial referencia a los trópicos. Tomo II: Predicción del rendimiento. FAO 22/2. Roma, Italia. 118 p.

BRUNER, H.D. e MOSER Jr., J.W., 1973. A Markov chain approach to the prediction of diameter distributions in uneven-aged forest stands. Can. J. For. Res. 3, 409-417.

BUONGIORNO, J. e MICHIE, B.R., 1980. A matrix model for uneven-aged forest management. For. Sci. 26: 609-625. U.S.A.

CLUTTER, J.L.; FORTSON, J.C.; PIENAAR, L.V.; BRISTER, G.H. e BAILEY, R.L., 1983. Timber management: a quantitative approach Ed. John Wiley \& sons New York, USA

DANIEL, P.W., HELMS, U.E. e BAKER, F.S., 1982. Principios de silvicultura. Ed Mc Graw Hill. México. 492 p.

DAVIS, L.S. e JOHNSON, K.N., 1987. Forest management. McGraw-Hill Book Co. USA. $790 \mathrm{p}$.

ENRIGHT, N. e OGDEN, J., 1979. Applications of transition matrix models in forest dynamics: Araucaria in Papua New Guinea and Nothofagus in New Zealand. Australian Journal of Ecology, n. 4, p. 3-23.

HARRISON, T. P. e MICHIE, B. R., 1985. A generalized approach to the use of matrix growth models. Forest Science, v. 31, n. 4, p. 850-856.

HOYOS, A., 1980. Processos estocásticos e previsão. In: $4^{0}$ Simpósio Nacional de Probabilidade e Estatística. Rio de Janeiro, 21 a 25 de julho de 1980.

LOWELL, K. E. e MITCHELL, R. J., 1987. Modeling growth and mortality probabilistically using logistic regression. USDA Forest Service NC GTR, St. Paul, p. 708-715.

MENDOZA, G. A. e SETYARSO, A., 1986. A transition matrix forest growth model for evaluating alternative harvesting schemes in Indonesia. Forest Ecology and Management., n. 15, p. 219-228. 
ROBERTS, M. R. e HRUSKA, A. J., 1986. Predicting diameter distributions: a test of the stationary Markov model. Can. J. For. Res., n. 16, p. 130-135.

SANQUETTA, C.R., 1996. Fundamentos biométricos dos modelos de simulação

florestal. FUPEF - Série didática № 08. Curitiba (PR), Brasil.

SPURR, S.H., 1952. Forest inventory. The Ronald Press Company. New York, USA. $476 \mathrm{p}$.

\section{ANEXOS}

Freqüências observadas $(1993 ; 1996)$ e simuladas (1999) com as matrizes de transição. Observed (1993;1996) and simulated (1999) with transition matrices frequencies.

\begin{tabular}{|c|c|c|c|c|c|c|c|c|c|c|c|c|c|c|c|c|c|c|}
\hline \multirow[t]{2}{*}{$\begin{array}{l}\text { Classe } \\
\text { Class }\end{array}$} & \multirow{2}{*}{$\begin{array}{l}\text { DAP } \\
D B H \\
{[\mathrm{~cm}]}\end{array}$} & \multicolumn{3}{|c|}{$\begin{array}{c}\text { Tratamento } 1 \\
\text { Treatment } 1\end{array}$} & \multirow{2}{*}{$\begin{array}{l}\text { DAP } \\
D B H \\
{[\mathrm{~cm}]}\end{array}$} & \multicolumn{3}{|c|}{$\begin{array}{c}\text { Tratamento } 2 \\
\text { Treatment } 3\end{array}$} & \multicolumn{3}{|c|}{$\begin{array}{c}\text { Tratamento } 3 \\
\text { Treatment } 3\end{array}$} & \multicolumn{3}{|c|}{$\begin{array}{c}\text { Tratamento } 4 \\
\text { Treatment } 4\end{array}$} & \multirow{2}{*}{$\begin{array}{l}\text { DAP } \\
D B H \\
{[\mathrm{~cm}]}\end{array}$} & \multicolumn{3}{|c|}{$\begin{array}{c}\text { Tratamento } 5 \\
\text { Treatment } 5\end{array}$} \\
\hline & & 1993 & 1996 & 1999 & & 1993 & 1996 & 1999 & 1993 & 1996 & 1999 & 1993 & 1996 & 1999 & & 1993 & 1996 & 1999 \\
\hline 1 & 1 & 0 & 0 & 0 & 1,5 & 0 & 0 & 0 & 5 & 0 & 0 & 4 & 0 & 0 & 2 & 0 & 0 & 0 \\
\hline 2 & 3 & 0 & 0 & 0 & 4,5 & 8 & 0 & 0 & 21 & 5 & 1 & 22 & 0 & 0 & 6 & 8 & 0 & 0 \\
\hline 3 & 5 & 28 & 7 & 2 & 7,5 & 79 & 48 & 29 & 32 & 5 & 1 & 35 & 13 & 3 & 10 & 45 & 11 & 1 \\
\hline 4 & 7 & 256 & 150 & 85 & 10,5 & 492 & 135 & 47 & 143 & 48 & 11 & 119 & 22 & 7 & 14 & 561 & 49 & 10 \\
\hline 5 & 9 & 520 & 271 & 147 & 13,5 & 1063 & 563 & 205 & 757 & 138 & 38 & 459 & 106 & 21 & 18 & 292 & 292 & 27 \\
\hline 6 & 11 & 1282 & 449 & 202 & 16,5 & 389 & 698 & 394 & 444 & 471 & 92 & 450 & 185 & 42 & 22 & 27 & 496 & 258 \\
\hline 7 & 13 & 997 & 905 & 359 & 19,5 & 24 & 460 & 565 & 63 & 556 & 356 & 79 & 485 & 152 & 26 & 0 & 76 & 411 \\
\hline 8 & 15 & 235 & 962 & 724 & 22,5 & 0 & 143 & 664 & 0 & 196 & 442 & 0 & 278 & 336 & 30 & 0 & 8 & 196 \\
\hline 9 & 17 & 21 & 442 & 896 & 25,5 & 0 & 0 & 143 & 0 & 32 & 376 & 0 & 53 & 387 & 34 & 0 & 0 & 27 \\
\hline 10 & 19 & 0 & 78 & 393 & 28,5 & 0 & 0 & 0 & 0 & 0 & 114 & 0 & 0 & 165 & 38 & 0 & 0 & 2 \\
\hline 11 & 21 & 0 & 21 & 232 & 31,5 & 0 & 0 & 0 & 0 & 0 & 16 & 0 & 0 & 26 & & & & \\
\hline 12 & 23 & 0 & 7 & 180 & 34,5 & 0 & 0 & 0 & 0 & 0 & 0 & 0 & 0 & 0 & & & & \\
\hline 13 & 25 & 0 & 0 & 36 & 37,5 & 0 & 0 & 0 & 0 & 0 & 0 & 0 & 0 & 0 & & & & \\
\hline 14 & 27 & 0 & 0 & 9 & & & & & & & & & & & & & & \\
\hline 15 & 29 & 0 & 0 & 2 & & & & & & & & & & & & & & \\
\hline 16 & 31 & 0 & 0 & 0 & & & & & & & & & & & & & & \\
\hline 17 & 33 & 0 & 0 & 0 & & & & & & & & & & & & & & \\
\hline 18 & 35 & 0 & 0 & 0 & & & & & & & & & & & & & & \\
\hline 19 & 37 & 0 & 0 & 0 & & & & & & & & & & & & & & \\
\hline 20 & 39 & 0 & 0 & 0 & & & & & & & & & & & & & & \\
\hline
\end{tabular}

